\title{
Implementación de la NIC 41 AGRICULTURA en el registro contable de las empresas agrícolas colombianas: Implicaciones y beneficios
}

\section{Implementation of the NIC 41 AGRICULTURE in the accounting record of the Colombia agricultural companies: Implications and benefits}

Frazier-Josep Corredor-Marroquín ${ }^{1}$

Artículo de investigación:

Fecha de recepción:

2019/11/04

Fecha de aceptación:

2019/12/16

Esta publicación se encuentra bajo licencia:

Creative Commons ReconocimientoNoComercialSinObraDerivada 4.0 Internacional

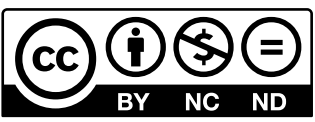

\section{Resumen}

Ante la falta de precisión en los métodos contables del Decreto 2649 de 1993 y de las demandas de un mercado cada vez más internacional y globalizado, resulta necesario modernizar y estandarizar los métodos contables de las empresas agrícolas colombianas. Esta modernización y adecuación a los estándares internacionales sirve para un incremento en la productividad, competitividad y rentabilidad de las empresas que lo adopten.

Las diversas investigaciones contables realizadas tanto en Colombia como en otros lugares de Latinoamérica en torno a la adopción del estándar NIC 41 determinan que su implementación es un gran avance para las empresas agrícolas. Dadas las implicaciones que se han demostrado en diversos cultivos agrícolas en diferentes territorios, una mayor capacitación de los empresarios y contadores para una adopción mayoritaria de esta norma en las empresas agrícolas del país supondría un aumento de la competitividad y fortalecería el tejido empresarial colombiano frente a los nuevos desafíos del mercado global.

Palabras claves: Contabilidad, agricultura, activos biológicos, NIC41, competitividad, palma de aceite.

Contador público, Especialista en Auditoría y Control, Facultad de Contaduría Pública - Universidad Santo Tomás, Docente tiempo completo, Frazier. corredor@Usantotomas.edu.co, código ORCID: https://orcid.org/00000002-0023-5008 


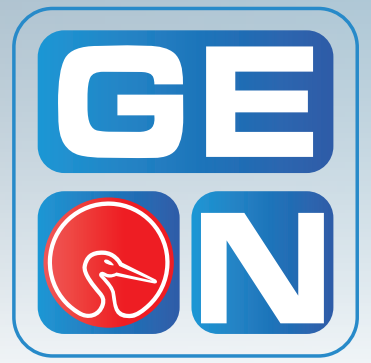

Revista GEON

(Gestión, Organizaciones y Negocios)

ISSN: 2346-3910 en línea

revistageon@unillanos.edu.co

Universidad de los Llanos

Colombia

Corredor Marroquin, F. (2020).

Implementación de la NIC 41 AGRICULTURA en el registro contable de las empresas agrícolas colombianas: Implicaciones y beneficios.

Revista GEON

(Gestión, Organizaciones y Negocios), 7(1), 142-164.

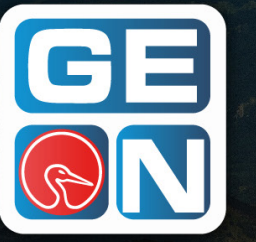

66 Las diversas investigaciones $f$ in 90 contables realizadas tanto en Colombia como en otros lugares de Latinoamérica en torno a la adopción del estándar NIC 41 determinan que su implementación es un gran avance para las empresas agrícolas. 99

Corredor Marroquin, F. (2020). Implementación de la NIC 41 AGRICULTURA en el registro contable de las empresas agrícolas colombianas: Implicaciones y beneficios.

Revista GEON (Gestión, Organizaciones Y Negocios), 7(1).

http://revistageon.unillanos.edu.co 


\section{Abstract}

In light of the lack of accuracy in the accountable methods regarding the Decree 2649 of 1993 and the demands of an international and globalized market, it's neccesary to modernize and standarize the accountable methods of the colombian agricultural companies. This modernization and adequacy to the international standards act as an increase in productivity, competitiveness and profitability to those companies that adopt it.

The different accountable researches made in Colombia as well as in other places all over Latin America in relation to the adoption of the standard NIC 41 determine that its implementation is a big step forward for the agricultural companies. Due to the proven implications in several agricultural crops in different territories, the entrepreneurs and accountants need greater training in this field in order to achieve a majority adoption of this standard. This would lead to an increase in competitiveness and would strengthen the colombian business network facing the new challenges of the global market.

Corredor Marroquin, F. (2020) Implementación de la NIC 41 AGRICULTURA en el registro contable de las empresas agrícolas colombianas: Implicaciones y beneficios. Revista GEON (Gestión, Organizaciones Y Negocios), 7(1) 142-164. https://doi. org/10.22579/23463910.189

Keywords: Accountability, agriculture, biological assets, NIC41, competititveness, oil palm

Códigos JEL: M10, M41

\section{Introducción}

Colombia posee una geografía favorable a los procesos agrícolas presentes en la región. Los terrenos aptos para cultivos son numerosos y su capacidad de crecimiento es aún mayor. Se calcula que, a pesar de tener 60 millones de hectáreas protegidas, de bosque y urbanas, hay 40 millones de hectáreas aptas para la agricultura de las cuales sólo 7.6 millones están aprovechadas.

Desde esta perspectiva, el campo colombiano cuenta con altas posibilidades de producción agrícola, no obstante el uso de suelos para cultivos ilícitos, la migración campesina y algunos fenómenos climáticos ralentizan y obstaculizan el desarrollo del mismo. 
Figura 1: Área sembrada estimada 1990-2014 (millones de hectáreas)

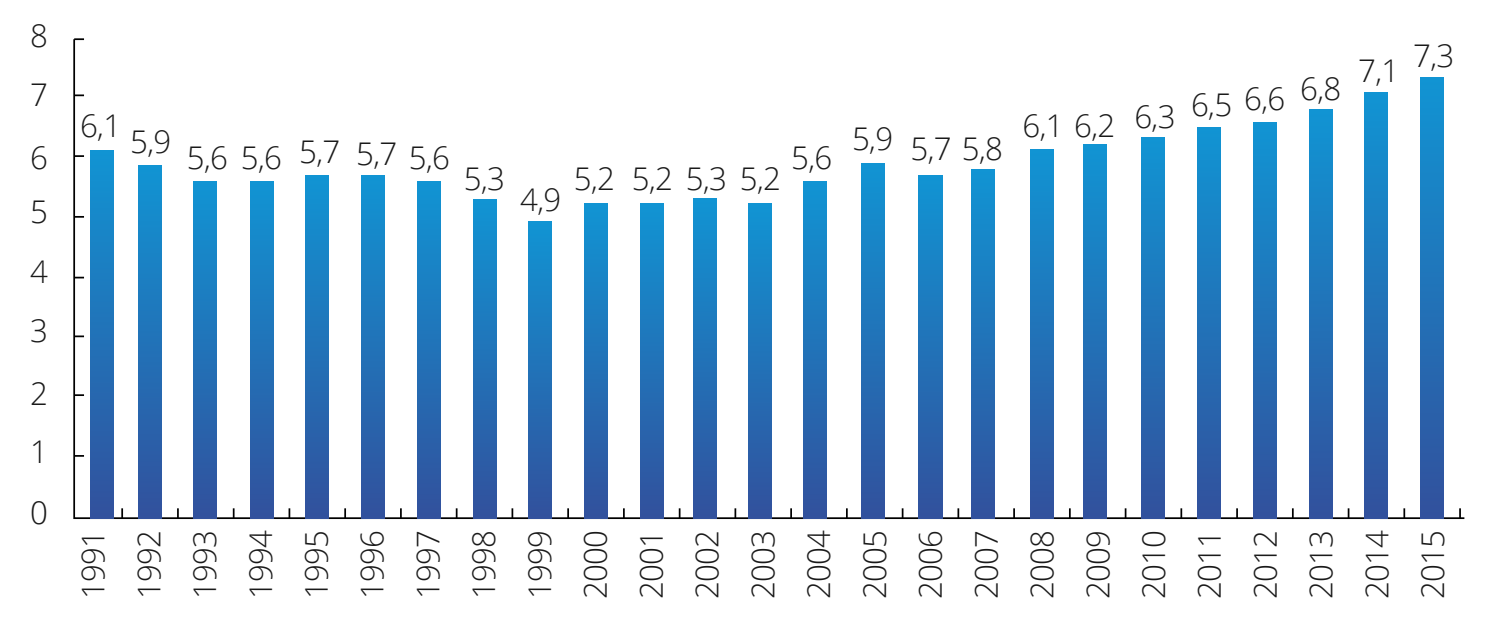

Fuente: MADR - DANE

La participación de Colombia en el mercado internacional de productos agrícolas de amplia y extensa producción en el país como el aguacate, el cacao, el banano, el maíz, el mango, la piña, etc. Es considerablemente bajo haciéndolo dependiente de las condiciones impuestas por el mercado global.

Se entiende por producción agrícola la cadena de actividades que genera una transformación biológica de plantas y animales para su comercialización. No obstante, es la población rural la encargada de poner a funcionar este sistema económico y sus desventajas no son pocas: hay bajo nivel de escolaridad, su infraestructura es bastante inferior a la urbana, tiene numerosas zonas de difícil acceso y, aún más preocupante, posee un alto índice de pobreza que repercute en el desarrollo de un país cuya industria depende en gran medida de su productividad rural.

Estas problemáticas tienen un impacto en el PIB del país, y en el porcentaje que la agricultura representa sobre el total. Si bien en los años 60 llegó a sobrepasar el 60\%, actualmente se sitúa en el $6 \%$. A pesar de que se observa un crecimiento en el primer trimestre del 2019 y en el conjunto de la última década, la situación del sector agrícola colombiano está lejos de ser ideal y el PIB Agrícola, en comparación con el PIB total, resulta aún muy bajo. Por ello, es necesario el aprovechamiento de todos los mecanismos posibles para el crecimiento y la valoración adecuada de los activos agrícolas del país. Uno de estos mecanismos es la adecuación del sistema contable de los activos biológicos a las normas internacionales de contabilidad con el objetivo de ser más competitivo y facilitar el comercio con países extranjeros. En concreto vamos a analizar el impacto de la norma NIC 41 en una empresa del Departamento del Meta y observaremos cómo resulta su contabilidad actual, bajo las normas internacionales de contabilidad. 
Tabla 1. Valor agregado por actividad económica

\begin{tabular}{|c|c|c|}
\hline \multicolumn{3}{|c|}{ Tasas de crecimiento en volumen ${ }^{2019^{p r}}$ - Primer trimestre } \\
\hline \multirow{4}{*}{ Actividad económica } & \multicolumn{2}{|c|}{ Tasas de crecimiento } \\
\hline & Serie original & $\begin{array}{l}\text { Serie corregida de efecto } \\
\text { estacional y calendario }\end{array}$ \\
\hline & Anual & Trimestral \\
\hline & 2019pr - I / 2018pr - I & 2019pr - I / 2018pr - IV \\
\hline Agricultura, ganadería, caza, silvicultura y pesca & 1,4 & 0,8 \\
\hline Explotación de minas y canteras & 5,3 & 2,2 \\
\hline Industrias manufactureras & 2,9 & $-1,0$ \\
\hline $\begin{array}{l}\text { Suministro de electricidad, gas, vapor y aire } \\
\text { acondicionado** }\end{array}$ & 3,1 & $-0,4$ \\
\hline Construcción & $-5,6$ & $-4,0$ \\
\hline Comercio al por mayor y al por menor*** & 4,0 & 1,4 \\
\hline Información y comunicaciones & 3,9 & $-1,7$ \\
\hline Actividades financieras y de seguros & 5,5 & 2,8 \\
\hline Actividades inmobiliarias & 3,0 & 0,7 \\
\hline Actividades profesionales, científicas y técnicas ${ }^{* * *}$ & 3,0 & 5,7 \\
\hline $\begin{array}{l}\text { Administración pública, defensa, educación y } \\
\text { salud } \star * \star \star \star\end{array}$ & 3,3 & $-0,9$ \\
\hline $\begin{array}{l}\text { Actividades artísticas, de entretenimiento y } \\
\text { recreación y otras actividades de servicios }\end{array}$ & 2,1 & 0,0 \\
\hline Valor agregado bruto & 2,8 & 0,0 \\
\hline $\begin{array}{l}\text { Total impuestos menos subvenciones sobre los } \\
\text { productos }\end{array}$ & 2,6 & 0,3 \\
\hline Producto Interno Bruto & 2,8 & 0,0 \\
\hline
\end{tabular}

pr preliminar

* Series encadenadas de volumen con año de referencia 2015.

** Suministro de electricidad, gas, vapor y aire acondicionado; distribución de agua, evacuación y tratamiento de aguas residuales, gestión de desechos y actividades de saneamiento ambiental.

*** Comercio al por mayor y al por menor; reparación de vehículos automotores y motocicletas; transporte y almacenamiento; alojamiento y servicios de comida.

**** Actividades profesionales, científicas y técnicas; actividades de servicios administrativos y de apoyo.

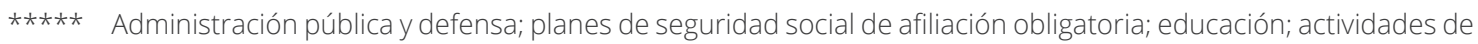
atención de la salud humana y de servicios sociales.

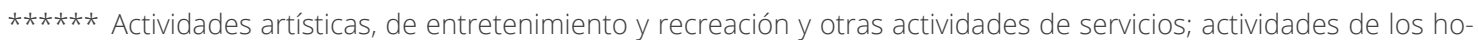
gares individuales en calidad de empleadores; actividades no diferenciadas de los hogares individuales como productores de bienes y servicios para uso propio.

Fuente: DANE, cuentas nacionales 


\section{Características y especificidades de la norma NIC 41}

Las Normas Internacionales de Información Financiera (NIIF) o (IFRS) son un conjunto de reglas que deben llevarse a la práctica a la hora de registrar la contabilidad de una empresa. La existencia de estas normas responde a la necesidad de estandarizar la contabilidad en un mundo globalizado para agilizar las transacciones financieras de empresas de diferentes naciones. Estos principios sirven para facilitar la verificación de los datos y para establecer un lenguaje financiero uniforme alrededor del mundo.

"En este sentido y en razón a las circunstancias como también a las nuevas exigencias que proponen estas normas de información contable y financiera, se fundaron organismos tales como International Accouting Standards Commite [IASC] junto con Standard Interpretations Comitee [SIC] en donde a partir del año 1973 trabajaron para crear estándares internacionales de contabilidad, popularmente conocidos como Normas Internacionales de Contabilidad [NIC]; posteriormente en el año 2001 se le dio paso a un nuevo emisor de estándares internacionales Ilamado International Accounting Standards Board [IASB] el cual surge con el fin de revisar, actualizar y modificar las pautas ya emitidas, creando así nuevos estándares denominados International Financial Reporting Standards [IFRS]" (Mayorga Murcia, 2018)

En este sentido, y debido a las profundas relaciones comerciales que mantiene Colombia en el ámbito de la agricultura, el país no se ha mantenido ajeno a esta corriente normativa y en el año 2009 el Gobierno colombiano aprobó la Ley 1314. El objetivo de esta ley era modernizar la contabilidad de las empresas, regida hasta entonces por el Decreto 2649 de 1993, y adecuarla a las NIIF (IFRS).

"La normatividad local expresada en el Decreto 2649 de 1993 manifiesta que dichos activos acordes a actividades agrícolas serán reconocidos por medio del costo histórico, aunque se puede presentar casos en donde el valor sea determinado por los precios del mercado o por aquellos establecidos fiscalmente por el gobierno, estas interpretaciones se pueden reflejar especialmente en el sector ganadero, además de eso, la norma no permite realizar disminuciones por ventas o gastos relacionados con el activo es aquí cuando se evidencia un impacto que claramente involucra los reportes emitidos por las empresas agropecuarias" (Mayorga Murcia, 2018)

De entre las normas recogidas por las NIIF este estudio se centrará en la Norma de Aplicación Contable (NIC) 41 "Agricultura", que es la norma que, a partir de la adopción de las NIIF, rige una parte sustancial de la contabilidad de las empresas agrícolas y, por ende, las de palma de aceite.

"NIC 41 establece el tratamiento contable de los activos biológicos a lo largo de su periodo de crecimiento, la valoración inicial de los productos agrícolas en el punto de su cosecha o recolección y las subvenciones oficiales. El objetivo de esta norma es 
regular el tratamiento contable, la presentación de estados financieros y la información a revelar de la actividad agrícola". (García Rivera, López Acosta, \& Cerón Espinosa, 2015, pág. 27)

"La NIC 41 prescribe, entre otras cosas, el tratamiento contable de los activos biológicos a lo largo del período de crecimiento, degradación, producción y procreación, así como la medición inicial de los productos agrícolas en el punto de su cosecha o recolección. Se requiere la medición de estos activos biológicos al valor razonable menos los costos estimados en el punto de venta, a partir del reconocimiento inicial realizado tras la obtención de la cosecha, salvo cuando este valor razonable no pueda ser medido de forma fiable al proceder a su reconocimiento inicial. Esta Norma se aplica a los productos agrícolas, que son los productos obtenidos de los activos biológicos de la entidad, pero sólo hasta el punto de su cosecha o recolección." (Fundación IRFS, 2012)

"Una entidad reconoce un activo biológico o la agricultura sólo cuando la entidad controla el activo como resultado de sucesos pasados, es probable que los beneficios económicos futuros vayan a fluir a la entidad, y el valor razonable o el costo del activo puede ser medido de forma fiable." (Consejo del IASC, 2000)

De entre los artículos más reseñables en lo que a diversos aspectos de la contabilidad se refiere la NIC 41 consideramos claves los siguientes para comprender las implicaciones que esta norma puede tener para la empresa:
Es importante señalar cómo los activos biológicos dentro del alcance de la NIC 41 se miden en el reconocimiento inicial y en posteriores fechas de referencia a su valor razonable, menos los costos estimados de venta, a menos que el valor razonable no se pueda medir de forma confiable. (NIC 41.12).

Cabe destacar de este apartado que se refiere a la obligatoriedad de una sucesiva medición del activo biológico, desde el inicio hasta el final del período contable, en diversas fechas de referencia, siempre a su valor razonable.

Además, se debe tener en cuenta que los productos agrícolas cosechados o recolectados que procedan de activos biológicos de una entidad se medirán a su valor razonable menos los costos de venta en el punto de cosecha o recolección. (NIC 41.13)

La medición del valor razonable de un activo biológico, o de un producto agrícola, puede verse facilitada al agrupar los activos biológicos o los productos agrícolas de acuerdo con sus atributos más significativos, como por ejemplo, la edad o la calidad. La entidad seleccionará los atributos que se correspondan con los usados en el mercado como base para la fijación de los precios. (NIC 41.15)

En relación a la contabilidad de las ganancias o pérdidas de los activos biológicos, la NIC 41 establece una serie de precisiones claras sobre la manera de llevar estos aspectos en el balance.

Las ganancias o pérdidas surgidas en el reconocimiento inicial de un activo biológico a su valor razonable menos 
los costos de venta y por un cambio en el valor razonable menos los costos de venta de un activo biológico deberán incluirse en la ganancia o pérdida neta del periodo en que aparezcan. (NIC 41.26)

Puede aparecer una pérdida, en el reconocimiento inicial de un activo biológico, porque es preciso deducir los costos de venta, al determinar el valor razonable menos los costos de venta del mencionado activo biológico. Puede aparecer una ganancia, tras el reconocimiento inicial de un activo biológico, por ejemplo, a causa del nacimiento de un becerro. (NIC 41.27)

Las ganancias o pérdidas surgidas por causa del reconocimiento inicial de un producto agrícola, que se lleva al valor razonable menos los costos de venta, deberán incluirse en la ganancia o pérdida neta del periodo en el que éstas aparezcan. (NIC 41.28).

En relación con la imposibilidad de medir el valor razonable de forma fiable, la NIC 41 establece lo siguiente:

Se presume que el valor razonable de un activo biológico puede medirse de forma fiable. Sin embargo, esa presunción puede ser refutada, sólo en el momento del reconocimiento inicial, en el caso de los activos biológicos para los que no estén disponibles precios cotizados de mercado, y para los cuales se haya determinado claramente que no son fiables otras mediciones alternativas del valor razonable. En tal caso, estos activos biológicos deben ser medidos a su costo menos la depreciación acumulada y cualquier pérdida acumulada por deterioro del valor. Una vez que el valor razonable de estos activos biológicos pase a medirse fiablemente, la entidad debe medirlos a su valor razonable menos los costos de venta. Una vez que el activo biológico no corriente cumple los criterios para ser clasificado como mantenido para la venta (o ha sido incluido en un grupo de activos para su disposición que ha sido clasificado como mantenido para la venta), de acuerdo con los criterios de la NIIF 5 Activos no Corrientes Mantenidos para la Venta y Operaciones Discontinuadas, se supone que el valor razonable puede ser medido con fiabilidad (NIC 41.30)

La presunción del párrafo 30 sólo puede ser rechazada en el momento del reconocimiento inicial. La entidad que hubiera medido previamente el activo biológico a su valor razonable menos los costos de venta, continuará haciéndolo así hasta el momento de la disposición. (NIC 41.31)

\section{Comparativa en relación al Decreto 2649 de 1993}

Las modificaciones que establece la NIC 41 con respecto al Decreto se van a enumerar en la tabla 2.

A raíz de esta comparativa se puede determinar que en el Decreto 2649 de 1993 los activos biológicos se pueden tratar como inventarios o como gastos cuando se incurren en ellos mientras que la NIC 41 determina claramente los requisitos para reconocer los activos biológicos como productos agrícolas. "En el Decreto 2649 los activos biológicos se miden al costo histórico. Sin embargo, para la actividad ganadera muchas veces 
Tabla 2: Diferencias entre el Decreto 2649 y el NIC-41

\begin{tabular}{|c|c|c|}
\hline & Decreto 2649 de 1993 & NIC-41 \\
\hline Art. 53 & $\begin{array}{l}\text { El manejo de costos permite aplazar los costos } \\
\text { de cultivos de tardío rendimiento, en cosechas } \\
\text { cuyo rendimiento es superior a } 2 \text { años. }\end{array}$ & $\begin{array}{l}\text { Los costos de producción y cosecha tienen que } \\
\text { ser añadidos en el balance en el momento en } \\
\text { que tengan lugar. }\end{array}$ \\
\hline \multirow[b]{3}{*}{ Art. 63} & $\begin{array}{l}\text { En referencia a la contabilidad de los activos, } \\
\text { éstos se pueden considerar como inventarios } \\
\text { o como gastos en el instante en que tiene lugar } \\
\text { la el reconocimiento. }\end{array}$ & $\begin{array}{l}\text { Los puntos que recogen qué es un activo agrí- } \\
\text { cola están especificados claramente hasta el } \\
\text { momento de cosecha o recolección. }\end{array}$ \\
\hline & $\begin{array}{l}\text { Los activos biológicos se toman como inven- } \\
\text { tario y son medidos según el coste histórico. }\end{array}$ & $\begin{array}{l}\text { El valor razonable es el instrumento usado para } \\
\text { su medición. }\end{array}$ \\
\hline & $\begin{array}{l}\text { La estipulación del valor de los activos tiene en } \\
\text { cuenta cargos directos, indirectosy erogaciones } \\
\text { que hayan tenido lugar para la finalización del } \\
\text { activo, ya dispuesto para su utilización o venta. }\end{array}$ & $\begin{array}{l}\text { El proceso de valoración debe ocurrir en el } \\
\text { momento de su reconocimiento inicial y en } \\
\text { otras fechas posteriores de medición. Se } \\
\text { tomará en cuenta el valor razonable menos } \\
\text { los costos de venta. Si el valor razonable no se } \\
\text { puede medir en el reconocimiento inicial no se } \\
\text { tendrá en cuenta. }\end{array}$ \\
\hline Art. 67 & $\begin{array}{l}\text { En el momento en que se la transacción del } \\
\text { activo tiene se reconoce la utilidad o pérdida } \\
\text { del mismo. }\end{array}$ & $\begin{array}{l}\text { El beneficio o la pérdida se deben reconocer } \\
\text { en la contabilidad cuando estos se realicen y } \\
\text { no cuando se reciba o se haga el pago. }\end{array}$ \\
\hline Art. 71 & $\begin{array}{l}\text { En relación a la capitalización de costos, se con- } \\
\text { sidera la suma todos los costos incurridos hasta } \\
\text { el momento de venta, incluidos los costos aso- } \\
\text { ciados y los de mantenimiento de los activos. }\end{array}$ & $\begin{array}{l}\text { Hasta que pueda contabilizarse el valor razo- } \\
\text { nable del activo se valoran todos los costos } \\
\text { menos la amortización sufrida por los mismos. }\end{array}$ \\
\hline Art. 73 & $\begin{array}{l}\text { El crédito tiene que estipularse como correc- } \\
\text { ción monetaria en el balance de manera pro- } \\
\text { porcional al costo del activo. }\end{array}$ & $\begin{array}{l}\text { La empresa deberá presentar los créditos } \\
\text { monetarios de forma separada por cada grupo } \\
\text { de activos biológicos durante el periodo. }\end{array}$ \\
\hline
\end{tabular}

Fuente: DANE, cuentas nacionales

se utilizan los valores que fiscalmente establecen el gobierno, los cuales son muy diferentes de sus valores reales. En otras ocasiones, por normas de la superintendencia de Sociedades, se debe valorar a precios de mercado sin establecer disminuciones de valores por gastos de ventas del mismo (...) Para la NIC 41 se utilizan el concepto de valor razonable con efecto en resultados. (...) Bajo el Decreto 2649 las utilidades o pérdidas solo se reconocen cuando se enajenan los productos mientras que en el NIC 41 mediante la aplicación del valor razonable se van reconociendo las ganancias o pérdidas aun cuando no se haya realizado la venta" (límenes Jaimes. 2005).

"En cuanto a la determinación del costo de producción y ventas en el Decreto 2649 / 93, se debe elaborar el estado financiero de resultados como estado financiero de propósito general y el de costos es adicional. En la NIC 41 Agricultura las afectaciones se hacen directamente al estado de resultados, generando cambios significativos en la utilidad operativa en cada una de estas situaciones." (Rodríguez García \& Ruiz Torres, 2016, pág. 196). 
La Norma NIC 41 en las empresas agrícolas colombianas: Estudio de caso e implicaciones.

Para un mejor acercamiento a lo que supone la aplicación de la NIC 41 a la contabilidad de las empresas agrícolas del Departamento del Meta realizaremos el estudio de caso y el análisis de los datos de la investigación llevada a cabo en la empresa Las Palmitas por parte de Sandy Tatiana Mendoza Gómez, investigadora de la Universidad Santo Tomás.

Las Palmitas poseen dentro de su cultivo las siguientes plantas:

10 plantas clase 1 de 3 años de edad

10 plantas clase 2 de 2 años de edad

Tabla 3: Medición inicial al valor razonable menos los costos de venta de las plantas

\begin{tabular}{c|c|c} 
Unidad & Detalle & Valor \\
\hline 10 & $\begin{array}{l}\text { Clase } 3-3 \text { años } \\
(10 * 2400.000)\end{array}$ & 24.000 .000 \\
\hline 10 & $\begin{array}{l}\text { Clase } 2-2 \text { años } \\
(10 * 2.000 .000)\end{array}$ & 20.000 .000 \\
\hline Costo total del activo biológico & 44.000 .000 \\
\hline Unidad & Detalle & Valor \\
\hline 10 & $\begin{array}{l}\text { Clase } 3-3 \text { años } \\
(10 * 2400.000)\end{array}$ & 24.000 .000 \\
\hline 10 & $\begin{array}{l}\text { Clase } 2-2 \text { años } \\
(10 * 2.000 .000)\end{array}$ & 20.000 .000 \\
\hline
\end{tabular}

Compra el 1 de Julio del 2018

\begin{tabular}{ccc}
\hline 1 & Clase $1-1$ año & 1.600 .000 \\
\hline 5 & Clase $0(5 * 300.000)$ & 1.500 .000
\end{tabular}

Costo total del activo biológico $\quad 47.100 .000$

\begin{tabular}{ccc} 
Unidad & Detalle & Valor \\
\hline 10 & Clase 3 & 28.000 .000 \\
\hline 10 & Clase 2 & 25.500 .000 \\
\hline 1 & Clase 1 & 2.000 .000 \\
\hline 5 & Clase 0 & 2.200 .000 \\
\hline \multicolumn{2}{c}{$\begin{array}{c}\text { Incremento total por los } \\
\text { cambios en el valor } \\
\text { razonable - Costo de venta. }\end{array}$} & $\mathbf{5 7 . 7 0 0 . 0 0 0}$ \\
\hline
\end{tabular}

Fuente: Elaboración de Sandy Tatiana Mendoza Gómez

Cálculo del valor estimable de un producto agrícola sobre la base del valor razonable menos costos de venta.

"La compañía Las palmitas, propietaria de una finca de palmas ha finalizado la recolección del fruto con un total de 100.000 kilos antes de ser colocado en el mercado realiza una limpieza en donde se elimina los restos de fruta que quedaron en el proceso de selección este proceso tuvo un costo que ascendió a 5.000.000. Según las estadísticas de la compañía los residuos suelen representar un 5\% del total de la recolección.

Luego se transporta al mercado activo con un costo de 2.000.000 la empresa normalmente, contrata un intermediario que se encarga de llevar a cabo todas las transacciones que se efectúen en el mercado con unos honorarios del $1 \%$ sobre el valor facturado, el dueño del edificio en donde se encuentra el mercado aplica una comisión del 2\%, sobre el valor de mercado del fruto. El precio de cotización en el mercado activo de dicho producto es de 1000/Kilo." (Mendoza Gómez. 2018) 
Tabla 4: Cálculo estimado a producto agrícola:

\begin{tabular}{|c|c|c|}
\hline Detalle & Parciales & Totales \\
\hline \multicolumn{2}{|c|}{$\begin{array}{l}\text { Valor de mercado del producto } \\
\text { agrícola (95.000 kilo *1000) }\end{array}$} & 95.000 .000 \\
\hline \multicolumn{2}{|c|}{$\begin{array}{l}\text { Costo estimado hasta el punto } \\
\text { de venta }\end{array}$} & -7000.000 \\
\hline Costo selección fruto & 5.000 .000 & \\
\hline Costo transporte & 2.000 .000 & \\
\hline \multicolumn{2}{|c|}{ Valor razonable del fruto } & 88.000 .000 \\
\hline \multicolumn{2}{|c|}{$\begin{array}{l}\text { Costos estimados en el punto de } \\
\text { venta }\end{array}$} & 2.850 .000 \\
\hline $\begin{array}{l}\text { Comisión 2\% dueño } \\
\text { edif. }\end{array}$ & 1.900 .000 & \\
\hline $\begin{array}{l}\text { Comisión } 1 \% \text { para } \\
\text { intermediario. }\end{array}$ & 950.000 & \\
\hline \multicolumn{2}{|c|}{$\begin{array}{l}\text { Valor razonable - costos } \\
\text { estimados en el punto de venta. }\end{array}$} & 85.150 .000 \\
\hline
\end{tabular}

Fuente: Elaboración de Sandy Tatiana Mendoza Gómez

Los productos agrícolas cosechados o recolectados de los activos biológicos de una entidad deben ser valorados, en el punto de cosecha o recolección, según su valor razonable menos los costes de venta. Tal valoración es el coste en esa fecha, cuando se aplique la NIC 2 Existencias u otra Norma que sea de aplicación (NIC 41. Sección 34. Párrafos 34.8 a 34.7 y 34.8 a 34.10).

La empresa PALMITAS se dedica a la plantación de palma, en el ejercicio de su actividad agrícola, a partir del 25 de Enero del 2018, ha realizado los siguientes desembolsos relacionados directamente con una plantación.

Al 1 de Marzo se considera que ya está listo el activo biológico para ser reconocido se estableció un valor razonable - costos de venta de $\$ 900.000 .000$.

Tabla 5: Desembolsos a partir del 25 de Enero de 2018

\begin{tabular}{lc}
\multicolumn{1}{c|}{ Detalle } & \multicolumn{1}{c}{ Costo } \\
\hline Preparación de terreno & 260.000 .000 \\
\hline Abono de terreno & 105.000 .000 \\
\hline Jornales de abono de terreno & 128.000 .000 \\
\hline Semillas & 179.000 .000 \\
\hline Jornales de siembra & 98.000 .000 \\
\hline Fungicidas & 45.000 .000 \\
\hline Jornales de aplicación de & 22.000 .000 \\
\hline fungicidas & 837.000 .000 \\
\hline Total costos & 36.500 .000 \\
\hline Abono para las plantas & 146.500 .000 \\
\hline Jornales de abono a las plantas & 183.000 .000 \\
\hline Desembolsos posteriores a 25 & \\
\hline de Marzo &
\end{tabular}

Fuente: Elaboración de Sandy Tatiana Mendoza Gómez

El 30 de Abril el valor del activo biológico a través del valor razonable menos los costos de venta fue estimado en $\$ 980.000 .000$.

Para el 30 de Junio fue nuevamente medido el cultivo encontrándose una estimación de \$1.150.000.000.

En la fecha del 31 de Julio se consideró que el cultivo ya estaba listo para cosecharse.

Al final en el mes de agosto se ha obtenido una producción de 100 Toneladas. El valor razonable menos costo de venta ha sido establecido en 13.000/Kilo. 
Tabla 6: Reconocimiento del costo del establecimiento del cultivo. (25 de Enero).

\begin{tabular}{ll}
\hline $\begin{array}{l}\text { Costo del activo biológico de } \\
\text { producción }\end{array}$ & 837.000 .000 \\
\hline $\begin{array}{l}\text { Inventario de materiales y } \\
\text { suministros }\end{array}$ & 837.000 .000 \\
\hline
\end{tabular}

Fuente: Elaboración de Sandy Tatiana Mendoza Gómez

Tabla 7: Registro del activo biológico al inicio del mismo. (1 de Marzo).

\begin{tabular}{lc}
\hline $\begin{array}{l}\text { Activo biológico de producción } \\
\text { por madurar }\end{array}$ & 900.000 .000 \\
\hline $\begin{array}{l}\text { Costo del activo biológico de } \\
\text { producción }\end{array}$ & 837.000 .000 \\
\hline $\begin{array}{l}\text { Utilidades por apreciación de } \\
\text { cultivos }\end{array}$ & 63.000 .000 \\
\hline
\end{tabular}

Fuente: Elaboración de Sandy Tatiana Mendoza Gómez

Los desembolsos realizados para el desarrollo del cultivo una vez este fue reconocido a su valor razonable menos los costos de venta deben ser llevados al GASTO, pues harán la disminución de la utilidad en el estado de resultados.

Tabla 8: Registro de desembolsos posteriores para el desarrollo del cultivo. (25 de Marzo).

\begin{tabular}{lr}
\hline Gasto de cultivos por madurar & 183.000 .000 \\
\hline Bancos & 183.000 .000 \\
\hline
\end{tabular}

Fuente: Elaboración de Sandy Tatiana Mendoza Gómez

Reconocimiento de cambios del valor del cultivo en las distintas fechas:

Tabla 9: 30 Abril. Transformación biológica del cultivo a valor razonable 980.000.000.

\begin{tabular}{ll}
\hline $\begin{array}{l}\text { Activo biológico de producción } \\
\text { por madurar }\end{array}$ & 80.000 .000 \\
\hline $\begin{array}{l}\text { Utilidades por apreciación de } \\
\text { cultivos }\end{array}$ & 80.000 .000 \\
\hline
\end{tabular}

Fuente: Elaboración de Sandy Tatiana Mendoza Gómez
Tabla 10: 30 Junio. Transformación biológica del cultivo a valor razonable 1.150.000.000

\begin{tabular}{ll}
\hline $\begin{array}{l}\text { Activo biológico de producción } \\
\text { por madurar }\end{array}$ & 170.000 .000 \\
\hline $\begin{array}{l}\text { Utilidades por apreciación de } \\
\text { cultivos }\end{array}$ & 170.000 .000 \\
\hline
\end{tabular}

Fuente: Elaboración de Sandy Tatiana Mendoza Gómez

Tabla 11: 31 Julio. El cultivo ha madurado (Debe ser trasladado de cultivo por madurar a maduro).

\begin{tabular}{ll}
\hline $\begin{array}{l}\text { Activo biológico de producción } \\
\text { maduro }\end{array}$ & 1.150 .000 .000 \\
\hline $\begin{array}{l}\text { Activo biológico de producción } \\
\text { por madurar }\end{array}$ & 1.150 .000 .000 \\
\hline
\end{tabular}

Fuente: Elaboración de Sandy Tatiana Mendoza Gómez

Tabla 12: Valoración económica de la producción de fruto de palma una vez recolectada la cosecha: 100 Toneladas * $(1$ Tonelada $=1000$ kilos $)=100.000$ Kilos * 13.000 Kilo: 1.300.000.000.

\begin{tabular}{lc}
\hline Producto agrícola & 1.300 .000 .000 \\
\hline $\begin{array}{l}\text { Activo biológico de producción } \\
\text { maduro }\end{array}$ & 1.150 .000 .000 \\
\hline $\begin{array}{l}\text { Utilidades por apreciación } \\
\text { de cultivos }\end{array}$ & 150.000 .000 \\
\hline
\end{tabular}

Fuente: Elaboración de Sandy Tatiana Mendoza Gómez

Los activos biológicos pueden ser clasificados como maduros o por madurar. Los activos biológicos maduros son aquellos que han alcanzado las condiciones para su cosecha o recolección (en el caso de activos biológicos consumibles), o son capaces de sostener producción, cosechas o recolecciones de forma regular (en el caso de los activos biológicos para producir frutos) - NIC 41. 34. 2

Las ganancias o pérdidas surgidas por causa del reconocimiento inicial 
de un activo biológico según su valor razonable menos los costes de venta, así como las surgidas por todos los cambios sucesivos en el valor razonable menos los costes de su venta, deben incluirse en la ganancia o pérdida del ejercicio contable en que aparezcan - NIC 41. 34. 4 a 34.7

Las ganancias o pérdidas surgidas por el reconocimiento inicial de un producto agrícola, que se contabiliza según su valor razonable menos los costes de venta, deben incluirse en la ganancia o pérdida del ejercicio en el que estas aparezcan - NIC 41. 34.8 a 34.10

Contabilización de subvenciones condicionadas para el establecimiento de cultivos.

La Gobernación del Meta para apoyar a los cultivadores de palma, estableció una subvención de 5.000.000, por hectárea efectivamente plantada, siempre y cuando se verifique un desarrollo de los cultivos superior al 75\% en los próximos 3 años.

Un productor de Acacias adquiere iniciando el año 2015 un terreno de 200 Hectáreas. En el año 2016 siembra en 90 Hectáreas un total de 15.000 plantas.

Finalizando el año 2018 la dirección de la UMATA, confirmo que 14.200 plantas de las sembradas se desarroIlaron satisfactoriamente. En promedio en una hectárea se siembran 167 árboles (ver tabla No. 13).

En el año 2018 se evidencia que la empresa ha cumplido con las condiciones establecidas por el ente gubernamental en razón de:
Tabla 13: *Subvención de gobierno por hectárea 5.000.000 * 90 hectáreas cultivadas

\begin{tabular}{lr} 
Bancos & 450.000.000* \\
\hline $\begin{array}{l}\text { Cuentas por pagar por subven- } \\
\text { ciones de gobierno }\end{array}$ & 450.000 .000 \\
\hline
\end{tabular}

Fuente: Elaboración de Sandy Tatiana Mendoza Gómez

Establecemos a cuantas hectáreas en producción corresponden a 14.200 plantas en desarrollo.

90 hectáreas* 14.200 plantas en desarrollo / 15.000 plantas cultivadas= 85,2 hectáreas aptas en cultivo, esto equivale al 94,66\% del desarrollo de los cultivos.

Tabla 14: Auxilio gubernamental 85,20 hectáreas * 5.000.000 $=426.000 .000$

\begin{tabular}{ll}
\hline $\begin{array}{l}\text { Cuentas por pagar por subven- } \\
\text { ciones de gobierno }\end{array}$ & $426.000 .000^{*}$ \\
\hline Ingresos por subvenciones & $426.000 .000^{*}$ \\
\hline
\end{tabular}

Fuente: Elaboración de Sandy Tatiana Mendoza Gómez

Los restantes 24.000.000 deben devolverse a la Gobernación del Meta.

Si una subvención oficial relacionada con un activo biológico que se valora a su valor razonable menos los costes de venta está condicionada, incluyendo situaciones en las que la subvención requiere que una entidad no emprenda determinadas actividades agrícolas, la entidad reconocerá la subvención oficial en el resultado del ejercicio cuando, y sólo cuando, se hayan cumplido las condiciones ligadas a ella (NIC, 41). 
Tabla 15: Balance

\begin{tabular}{|c|c|c|c|c|c|c|c|}
\hline & \multicolumn{7}{|c|}{ Parámetros } \\
\hline & Año 1 & Año 2 & Año 3 & Año 4 & Año 5 & Año 6 & Año 7 \\
\hline Establecimiento \$/ha & \multicolumn{7}{|l|}{12} \\
\hline Mantenimiento \$/ha & & 10 & 8 & 5 & 5 & \multicolumn{2}{|l|}{5} \\
\hline Cosecha $\$ /$ ha & & & & & & \multicolumn{2}{|r|}{20} \\
\hline Tasa Crecimiento Madera & $0 \%$ & $0 \%$ & $0 \%$ & $10 \%$ & $10 \%$ & $10 \%$ & $10 \%$ \\
\hline Toneladas Madera (Ton/ha) & & & 20 & 22,0 & 24,2 & 26,6 & 29,3 \\
\hline Tasa Inflación & & $3 \%$ & $3 \%$ & $3 \%$ & $3 \%$ & $3 \%$ & $3 \%$ \\
\hline Precios (\$/Ton) & 3,0 & 3,1 & 3,2 & 3,3 & 3,4 & 3,5 & 3,6 \\
\hline Ha a Plantar & 20.000 & & & & & & \\
\hline Costos & 240.000 & 200.000 & 160.000 & 100.000 & 100.000 & 100.000 & 400.000 \\
\hline \multicolumn{8}{|c|}{ Medición } \\
\hline Valor de Mercado & - & - & 1.273 .080 & 1.442 .400 & 1.634 .239 & 1.851 .593 & 2.097 .854 \\
\hline Costo Acumulado & 240.000 & 440.000 & & & & & \\
\hline \multicolumn{8}{|c|}{ Reconocimiento } \\
\hline Plantaciones S I & - & 240.000 & 440.000 & 1.273 .080 & 1.442 .400 & 1.634 .239 & 1.851 .593 \\
\hline Adiciones & 240.000 & 200.000 & 160.000 & 100.000 & 100.000 & 100.000 & 400.000 \\
\hline Ajuste al FV & & & 673.080 & 69.320 & 91.839 & 117.354 & $(153.738)$ \\
\hline Saldo Final & 240.000 & 440.000 & 1.273 .080 & 1.442 .400 & 1.634 .239 & 1.851 .593 & 2.097 .854 \\
\hline Plantaciones - Adiciones & 240.000 & 200.000 & 160.000 & 100.000 & 100.000 & 100.000 & 400.000 \\
\hline Plantaciones - Ajuste al FV & - & - & 673.080 & 69.320 & 91.839 & 117.354 & $(153.738)$ \\
\hline Ingreso (Egreso) Ajuste al FV & - & - & $(673.080)$ & $(69.320)$ & $(91.839)$ & $(117.354)$ & 153.738 \\
\hline Bancos & $(240.000)$ & $(200.000)$ & $(160.000)$ & $(100.000)$ & $(100.000)$ & $(100.000)$ & $(400.000)$ \\
\hline
\end{tabular}

Fuente: Elaboración de Sandy Tatiana Mendoza Gómez 
Tabla 16: Balance

\begin{tabular}{|c|c|c|c|c|c|c|c|}
\hline & \multicolumn{7}{|c|}{ Parámetros } \\
\hline & Año 1 & Año 2 & Año 3 & Año 4 & Año 5 & Año 6 & Año 7 \\
\hline Establecimiento \$/ha & \multicolumn{7}{|l|}{12} \\
\hline Mantenimiento \$/ha & & 10 & 8 & 5 & 5 & \multicolumn{2}{|l|}{5} \\
\hline \multicolumn{7}{|l|}{ Cosecha \$/ha } & 20 \\
\hline Tasa Crecimiento Madera & $0 \%$ & $0 \%$ & $0 \%$ & $10 \%$ & $10 \%$ & $10 \%$ & $10 \%$ \\
\hline \multicolumn{3}{|l|}{ Toneladas Madera (Ton/ha) } & 20 & 22,0 & 24,2 & 26,6 & 29,3 \\
\hline \multicolumn{2}{|l|}{ Tasa Inflación } & $3 \%$ & $3 \%$ & $3 \%$ & $3 \%$ & $3 \%$ & $3 \%$ \\
\hline Precios (\$/Ton) & 3,0 & 3,1 & 3,2 & 3,3 & 3,4 & 3,5 & 3,6 \\
\hline Ha a Plantar & \multicolumn{7}{|l|}{20.000} \\
\hline \multicolumn{8}{|c|}{ Flujo del proyecto } \\
\hline Ingresos & - & - & 1.273 .080 & 1.442 .400 & 1.634 .239 & 1.851 .593 & 2.097 .854 \\
\hline Costos & 240.000 & 440.000 & & & & & \\
\hline \multicolumn{8}{|l|}{ Flujo Neto } \\
\hline \multicolumn{8}{|c|}{ Medición } \\
\hline Tasa Descuento & \multicolumn{7}{|l|}{$15 \%$} \\
\hline VPN Flujos Futuros & 266.489 & 506.462 & 742.431 & 953.796 & 1.196 .865 & 1.476 .395 & 1.697 .854 \\
\hline \multicolumn{8}{|c|}{ Reconocimiento } \\
\hline Plantaciones S I & - & 266.489 & 506.462 & 742.431 & 953.796 & 1.196 .865 & 1.476 .395 \\
\hline Adiciones & 240.000 & 200.000 & 160.000 & 100.000 & 100.000 & 100.000 & 400.000 \\
\hline Ajuste al FV & 26.489 & 39.973 & 75.979 & 111.365 & 143.069 & 179.530 & $(178.541)$ \\
\hline Saldo Final & 266.489 & 506.462 & 742.431 & 953.796 & 1.196 .865 & 1.476 .395 & 1.697 .854 \\
\hline Plantaciones - Adiciones & 240.000 & 200.000 & 160.000 & 100.000 & 100.000 & 100.000 & 400.000 \\
\hline Plantaciones - Ajuste al FV & 26.489 & 39.973 & 75.979 & 111.365 & 143.069 & 179.530 & $(178.541)$ \\
\hline Ingreso (Egreso) Ajuste al FV & $(26.489)$ & (39.973) & $(75.979)$ & $(111.365)$ & (143.069) & $(179.530)$ & 178.541 \\
\hline Bancos & $(240.000)$ & $(200.000)$ & $(160.000)$ & $(100.000)$ & $(100.000)$ & $(100.000)$ & $(400.000)$ \\
\hline
\end{tabular}

Fuente: Elaboración de Sandy Tatiana Mendoza Gómez 
Como podemos ver en el balance, así como en los gráficos anteriores, la medición de los activos biológicos según su valor razonable en las diferentes etapas de crecimiento de la cosecha hace que los resultados finales, no sólo reflejen con mayor precisión el valor real de los activos de la empresa, sino que muestra con mayor precisión el valor de mercado de dichos activos. De esta manera vemos cómo el valor de mercado aumenta paulatinamente conforme los activos se van desarrollando de forma natural y esto hace que la empresa pueda ajustar los precios del producto final, haciendo que gane en competitividad. De la misma forma, esta manera de plasmar el balance hace que de cara a una posible expansión internacional su credibilidad y fiabilidad como empresa sea mayor, lo cual abre unas posibilidades que no existirían si se siguiera realizando la contabilidad de la forma anterior a la ley 1314 de 2009. Este ejemplo ilustra a la perfección el camino que deberían seguir las empresas agrícolas del Departamento del Meta a la hora de realizar su tratamiento contable ya que los beneficios de esta norma son manifiestos.

Cabe destacar que a pesar de que, como se ha visto, las diferencias entre la ley anterior y la NIC 41 son notables, aun con su paulatina implementación, existen aspectos a mejorar para impulsar la competitividad y la fiabilidad de las empresas agrícolas colombianas desde su sistema contable. Una de estas carencias viene dada por la ausencia de medición del impacto ambiental de estas empresas y cómo ese impacto repercute en la produc- ción. "El uso de prácticas inapropiadas en el sector, el desplazamiento de importantes áreas de cultivos de base alimentaria y la expansión progresiva de monocultivos que retan la biodiversidad, que sin duda impiden que el sector sea sostenible.

La sostenibilidad del sector por ahora está en entredicho, pues si bien, están realizando importantes esfuerzos por intermedio Fedepalma y organizaciones sin ánimo de lucro, no se reconocen sus esfuerzos, pues la sostenibilidad ambiental se evidencia en la medida que se reconozca el valor aproximado de los impactos.

Es necesaria la implementación de sistemas de información pertinentes con informes sobre la sostenibilidad y el valor del negocio, en lo cual es imperativo la adopción de procesos de aseguramiento de información y trasparencia contable." (Mendoza Gómez)

\section{Contexto Teórico.}

\section{La palma africana.}

Esta planta se define como:

La palma de aceite (elaeis guineensis) es una planta oleaginosa cuyo tallo puede alcanzar más de 30 metros de altura y produce racimos de frutos compactos que pueden pesar entre 10 y 40 kilogramos. Se caracteriza por ser la oleaginosa más productiva del planeta: en promedio, una hectárea sembrada produce entre seis y diez veces más aceite que otras. Aunque la vida productiva de la palma es mayor a cincuenta años, la altura que alcanza a los 20 o 25 años dificulta las labores 
de cosecha, lo cual indica el comienzo de la renovación en las plantaciones comerciales (Superintendencia de Industria y Comercio, pág. 1).

\section{La Palma Africana en Colombia.}

Fue traída a Colombia por Florentino Claes en 1932 y cultivada comercialmente hacia 1945 en el Departamento del Magdalena por United Fruit Company, es un cultivo que se ha expandido lentamente en el país sin embargo se dispone de 3,5 millones de hectáreas aptas (FEDEPALMA, s.f.)

La Federación Nacional de Cultivadores de Palma de Aceite Fedepalma señala que:

Colombia es el cuarto productor de aceite de palma en el mundo y el primer productor en América. La palma de aceite está presente en cuatro zonas de Colombia: Norte, Oriental, Central y Suroccidente, que abarcan 122 municipios en 19 departamentos alrededor del país, siendo su área sembrada de 500.000 hectáreas aproximadamente. La palmicultura es una de las actividades agrícolas más prometedoras como eje para alcanzar el desarrollo nacional (FEDEPALMA).

\section{La Palma Africana en el Meta}

De acuerdo al boletín económico de FEDEPALMA se muestra que la zona Oriental de Colombia a fecha de corte de Febrero del 2016 es la de mayor producción con respecto a otras regiones, con un acumulado del $41 \%$ de la producción representada en 525.000 toneladas de aceite de palma, 97000 toneladas de almendra de palma y un total de 181.543 hectáreas usadas para el desarrollo y producción de aceite de palma (FEDEPALMA, 2016).

\section{Normativa contable:}

\section{Decreto 2649 de 1993.}

En el Decreto 2649 de 1993 se definen los Principios de Contabilidad Generalmente Aceptados (PCGA) en Colombia, este detalla el ámbito de aplicación, objetivos, cualidades de la información, normas básicas y elementos de los estados financieros, su carácter es obligatorio por tratarse de una norma de tipo legal (Gobierno Nacional de la Republica de Colombia, 1993).

Los Principios de Contabilidad Generalmente Aceptados en Colombia son "El conjunto de conceptos básicos y de reglas que deben ser observados al registrar e informar contablemente sobre los asuntos y actividades de personas naturales o jurídicas" (Gobierno Nacional de la Republica de Colombia, 1993).

En este Decreto se indica que la contabilidad "Permite identificar, medir, clasificar, registrar, interpretar, analizar, evaluar, e informar, las operaciones de un ente económico en forma clara, completa y fidedigna" (Gobierno Nacional de la Republica de Colombia, 1993).

El ámbito de aplicación de este Decreto será para todas las personas que están obligadas a llevar contabilidad (Gobierno Nacional de la Republica de Colombia, 1993). 


\section{Colombia bajo Normas Internacionales de Información Financiera.}

Con el proceso de globalización y los tratados que realiza Colombia se emitió la Ley 1314 del 2009 con el fin de introducir al país en la dinámica financiera mundial. El objetivo de esta Ley ayuda a atraer inversión extranjera dinamizando el desarrollo del país y generando empleo con el fin de obtener mayor calidad y credibilidad en el mercado mundial. Actualmente el Consejo Técnico de la Contaduría Pública (CTCP) es el encargado de la coordinación del proceso de convergencia de estándares internacionales con participación de las diferentes entidades del Estado. (Acerca de nosotros: R\&R MORENO Auditores \& Consultores, s.f.)

Con la Ley 1314 de 2009 Colombia decide adoptar las NIIF/IFRS emitidas por el IASB.

El International Accounting Standards Board (IASB) es:

Un organismo independiente del sector privado que desarrolla y aprueba las Normas Internacionales de Información Financiera. El IASB funciona bajo la supervisión de la Fundación del Comité de Normas Internacionales de Contabilidad (IASCF). El IASB se constituyó en el año 2001 para sustituir al Comité de Normas Internacionales de Contabilidad (International Accounting Standards Committee) (Acerca de nosotros: NIC NIIF).

Las Normas Internacionales de Información Financiera (NIIF), emitidas por el IASB, son un grupo de estándares técnicos que especifican los parámetros para el reconocimiento, medición, presentación y revelación de información financiera en los estados financieros de propósito general de una organización (Sarmiento \& CTCP, 2014).

Las NIIF no sólo inciden en el ámbito contable y financiero sino que además se encuentran reflejadas desde la gerencia, pues debe existir un alto grado de compromiso por cada uno de los miembros de las organizaciones para que se logre iniciar con éxito esta conversión.

Las empresas que inicien con la aplicación de las NIIF deben estar catalogadas en tres grupos de la siguiente manera Grupo 1, Grupo 2, Grupo 3. (Bernal Chaparro, 2013)

Los grupos para la aplicación de NIIF en Colombia se acogerán a los decretos reglamentarios emitidos por el Gobierno Nacional. El grupo 1 por los Decretos 2784 del 2012, 3023 del 2013, 3024 del 2013 y 2615 del 2014; el grupo 2 por los Decretos 3022 del 2013, 2129 del 2014 y 2267 del 2014 y para el Grupo 3 los Decretos 2706 del 2012 y el 3019 del 2013.

A partir de la entrada en vigencia de las NIIF en Colombia, el proceso de adopción es una señal de la evolución que nuestro país está teniendo, en el cuál es primordial la estandarización del lenguaje contable para realizar estados financieros que reflejen la veracidad y transparencia corporativa (Olave, 2013). 


\section{Norma Internacional de Contabilidad 41.}

Esta norma es de vital importancia puesto que es la base que establece aspectos a desarrollar en el tratamiento contable de la actividad agrícola y en especial de los cultivos de palma africana.

La Norma Internacional de Contabilidad (NIC) 41 expresa el tratamiento contable que se debe realizar en la presentación de los estados financieros en la actividad agrícola, es un estándar que fue aprobado por el International Accounting Standards Committee (IASC) en Diciembre del 2000 y cuya vigencia se realiza para los estados financieros elaborados a partir del 1 de Enero del 2003, el objetivo de este estándar es detallar el tratamiento contable, la presentación en los estados financieros y la información a revelar relacionados con la actividad agrícola (IASCF, págs. 1,2).

Sin embargo es importante aclarar que el IASB público enmienda a la NIC 16 y 41 especialmente para las plantas (IFRS, 2014).

La NIC 41 define la actividad agrícola como "La gestión por parte de una empresa de la trasformación biológica de animales vivos o plantas ya sea para su venta, para generar productos agrícolas o para obtener activos biológicos adicionales" (IASCF, pág. 2).

El alcance de esta norma aplica para contabilizar factores relacionados con la actividad agrícola específicamente para los activos biológicos (IASCF).

El estándar define aspectos importantes para la Norma en mención; principalmente se establece que el producto agrícola es aquel producto que ya ha sido recolectado y es procedente de los activos biológicos de la empresa; otro concepto que especifica el estándar es el de activo biológico que puede ser el animal vivo o una planta, así mismo indica que la transformación biológica es aquella que comprende diversos procesos (crecimiento, degradación, producción y procreación) causa de los cambios cualitativos y cuantitativos en los activos biológicos; y finalmente precisa la definición de la cosecha o recolección que es la separación del producto del activo biológico del que procede o bien el cese de los procesos vitales de un activo biológico (IASCF, pág. 5).

Esta norma no aplica para terrenos ni para los activos intangibles que se relacionan con la actividad agrícola ya que estos tendrán un tratamiento contable diferente bajo otros estándares (IASCF, pág. 5).

\section{Materiales y Métodos.}

El desarrollo de esta investigación se basara en los siguientes instrumentos:

- Entrevistas y cuestionario estructurado a miembros de la empresa seleccionada para la investigación, con el objetivo de conocer el manejo actual de los procesos internos en áreas específicas.

- Diarios de campo de las visitas adelantadas en las oficinas contables de la empresa y de ser requerido, visitas a los cultivos de propiedad de empresa.

- Análisis documental del estándar internacional contable así como la normatividad contable de acuerdo al Decreto 2649 de 1993. 


\section{Resultados y Discusión}

En base a las investigaciones realizadas en el Departamento del Meta, en Socorro, Bucaramanga, así como otras realizadas por investigadores en Ecuador y Argentina se observa un patrón de resultados que permite inferir que una adopción definitiva y mayoritaria de la Norma NIC 41 revertirá en una mayor competitividad del sector agrícola colombiano. De las siguientes publicaciones

- Villacrés M.X., Aplicación de la NIC 41 (Agricultura) en la finca productora de Palmito "La Unión", ubicada en la provincia de Pichincha, Cantón Pedro Vicente Maldonado, Sector Paraíso Escondido. Quito. 2013. Pontificia Universidad Católica del Ecuador.

- Altuve A.R., Aplicación de la norma de contabilidad internacional NIC 41 Agricultura en el cultivo de cacao. Caso simulado en el municipio de Rionegro, Santander. Bucaramanga. 2016. Universidad Cooperativa de Colombia.

- Murillo T.R., La contametría en el proceso de implementación de la NIC 41 en actividades cafeteras, caso Finca Los Mandarinos, Socorro. Universidad Libre. Socorro.

Sin embargo se destaca que debido a los cambios del decreto 2649 del año 1993, por el mercado y la evolución de la economía global, cabe destacar que un retraso en la implementación de las NIIF supondría una dificultad para las empresas agrícolas colombianas en lo referente a su competitividad y por tal motivo la importancia de conocer los impactos y las implicaciones de la IAS 41 aplicada a los cultivos de palma africana.

\section{Conclusiones}

La adopción de las NIIF es esencial para el buen funcionamiento de las empresas agrícolas del Meta ya que, en comparación con el Decreto 2649 de 1993, se aproxima mucho más a la realidad de la empresa. Aspectos como el estudio de los gastos o la evaluación del inventario son mucho más precisos y ayudan a una mejor planificación, lo que favorece no sólo la gestión de las empresas sino la atracción para la inversión extranjera y la exportación de los productos agrícolas colombianos. Por ello, la adopción de la Norma NIC 41 mejora la anterior normativa y las empresas del Departamento del Meta deben ser persuadidas para que la implementen en sus registros, ya que más allá de la ley 1314 de 2009, el beneficio estratégico para ellas es notorio.

El hecho de que en el Decreto 2649 de 1993 prevalezca el registro histórico frente al valor razonable en el transcurso del crecimiento del activo supone una rémora para el cálculo real de los activos de la empresa. Debido a esto los intercambios económicos distan de su auténtico valor, llegando a distorsionar el balance.

Relacionado con esto, en lo referente a la capitalización de costos se presenta una problemática similar, ya que el Decreto no tiene en cuenta las amortizaciones hasta el momento en que se puede tener en cuenta el valor razonable, algo que sí tiene en cuenta 
la NIC 41, que también contempla en su medición los aspectos cualitativos y cuantitativos de los activos mientras que el Decreto sólo considera los cuantitativos. Además, la NIC 41 impide diferir el cargo a gastos en cultivos de rendimiento tardío, algo que sí permite el Decreto.

Si bien el Decreto 2649 de 1993 era una ley necesaria y pertinente en el momento en que se sancionó, actualmente, con las modificaciones sufridas por el mercado y la evolución de la economía global, cabe destacar que un retraso en la implementación de las NIIF supondría una dificultad para las empresas agrícolas colombianas en lo referente a su competitividad. Por todo lo expuesto en este estudio, recomendamos encarecidamente la adopción de este sistema de contabilidad internacional lo antes posible. Cuanto más se demore en tomar estas medidas, más pérdidas sufrirá la economía del sector y del país.

\section{Referencias}

Altuve A.R (2016) Aplicación de la norma de contabilidad internacional NIC 41 Agricultura en el cultivo de cacao. Caso simulado en el municipio de Rionegro, Santander. Bucaramanga. 2016. Universidad Cooperativa de Colombia. Obtenido de https://repository.ucc.edu. co/bitstream/20.500.12494/1752/1/ tratamiento\%20contable\%20 NIC\%20 41\%20Agricultura.pdf

Bernal Chaparro, C. A. (2013). Material pedagógico Ley de convergencia, Grupo de Investigación y Regulación Contable Superintendencia de Sociedades, Obtenido https://www.supersociedades.gov.co/delegatura_aec/ regulacion_contable/taller_grupo1/ Presentacion_1_Supersociedades.pdf

Calvo de Ramírez, A. (1). NIC 41: Tratamiento contable de los activos biológicos y los productos agrícolas. Contabilidad Y Auditoría, (21), 12 Págs. Recuperado a partir de http://www. ojs.econ.uba.ar/index.php/Contyaudit/article/view/127

Céspedes Vargas, M., Rodríguez Rodríguez, P., Franco, O. L., \& Tobar Martínez, J. (2018). Nivel de implementación de las NIIF en las funerarias de Villavicencio - Colombia. Revista GEON (Gestión, Organizaciones Y Negocios), 5(1), 42-53. https://doi. org/10.22579/23463910.12

Consejo del IASC. (2000). Norma Internacional de Contabilidad $n^{\circ} 41$ (NIC 41). www.normasinternacionalesdecontabilidad.es/nic/pdf/NIC41.pdf

Consejo Técnico de la Contaduría Pública (2015). Concepto - Medición de activos biológicos e inventarios en los cultivos de palma. Obtenido de http://cdn.actualicese.com/normatividad/2015/Conceptos/C397-15.pdf

Estrella Morales, A. (2015). IASB publica enmiendas a la NIC 16 y la NIC 41 para las plantas portadoras, Instituto Nacional de Contadores Públicos, Boletín 113, 12-15, https://incp.org.co/ Site/2015/publicaciones/boletin/boletin-incp-113.pdf

FEDEPALMA (2016). Balance económico del sector palmero colombiano en 2015, Boletín Económico Fedepalma. Obtenido de http://web.fedepalma. org/sites/default/files/files/BTE\%20 2016_en\%20baja.pdf

FEDEPALMA. (2011). Palma de aceite en Colombia: Federación Nacional de Cultivadores de Palma de Aceite. Obtenido de FEDEPALMA Web site: 
http://www.palmadeaceite.org/palma-de-aceite-en-colombia

FEDEPALMA. (s.f.). Historia de la Palma de Aceite Federación Nacional de Cultivadores de Palma de Aceite. Obtenido de http://www.palmadeaceite.org/historia-de-la-palma-de-aceite

Forero L. Y. y Velásquez V. A. (2008), Tratamiento de los activos biológicos en Colombia aplicando la NIC 41 (Agricultura). Bogotá. Universidad La Salle obtenido de https://ciencia.lasalle. edu.co/contaduria_publica/477/

García Rivera, K., López Acosta, M. L., \& Cerón Espinosa, H. (2015) Efecto de la NIC 41 en la actividad agrícola en Colombia. Magazín Empresarial, 11 (28), 23-32 obtenido de https://repository.usc.edu.co/bitstream/20.500.12421/2499/1/Efecto $\% 20$ de $\% 201$ a $\% 20$ NIC $\% 2041 \% 20$ en\%20la\%20actividad\%20agr\%C3\%ADcola\%20en\%20Colombia.pdf

IFRS Foundation. (2015) Normas internacionales de información financiera, primera edición. Obtenido de https://www.ifrs.org/ news-and-events/2018/04/normas-niif-2018-nuevos-libros-disponibles-en-espanol/

Jímenes Jaimes N.A., De las normas contables colombianas a las normas estándares - Internacionales de Información Financiera, Edición AGN Internacional. Pág. 78-250 obtenido de https://books.google.com.co/books/ about/De_las_normas_contables_coIombianas_a_la.html?id=pG|ZjwEACAAJ\&redir_esc $=y$

Joven, J.C. (2017), Tratamiento contable de las palmas de aceite en las NIIF para las empresas en Colombia. Bogotá. 2017. Universidad Militar Nueva Granada. Obtenido de https://repository.unimilitar.edu.co/bitstream/
handle/10654/17844/JovenTuberquiajessicaCarolina2017.pdf;jsessionid=E67296919A43847164AEED1BE5BA6E9B?sequence $=3$

Mayorga Murcia V.C., (2018) Impacto de la NIC 41 en el Sector agrícola colombiano. Bogotá. Universidad Militar Nueva Granada. Obtenido de https://repository.unimilitar.edu.co/ bitstream/handle/10654/18157/MayorgaMurciaVivianaCarolina2018.pdf? sequence=3

Mendoza Gómez S.T. (2018), El aseguramiento de la información en el cultivo sostenible de palma de aceite. Bucaramanga. Universidad Santo Tomás obtenido de http:// scienti.colciencias.gov.co:8081/cvlac/ visualizador/generarCurriculoCv.do?cod_rh=0001551023

Mendoza Gómez S.T. (2018), Tratamiento contable de activos biológicos en la actividad palmicultora. Villavicencio. Universidad Santo Tomás obtenido de http://scienti.colciencias.gov.co:8081/ cvlac/visualizador/generarCurriculoCv.do?cod_rh=0001551023

Murillo T.R \& Gualdrón, A. (2016), La contametría en el proceso de implementación de la NIC 41 en actividades cafeteras, caso Finca Los Mandarinos, Socorro. Universidad Libre. Socorro. Disponible en http://www.unilibre. edu.co/bogota/pdfs/2016/ponencias/7.pdf

NIC NIIF. (s.f.). Qué es el IASB Obtenido http://www.nicniif.org/home/iasb/ que-es-el-iasb.html

Olave, J. C. (2013). La adopción de las NIIF en Colombia, Revista Portafolio, obtenido de http://www.portafolio.co/opinion/juan-carlos-olave/adopcion-niif-colombia-76182

Organización de las Naciones Unidas para la Alimentación y la Agricultura. 
(2003). Agricultura mundial: hacia los años 2015/2030. Disponible en http:// www.fao.org/docrep/004/y3557s/ y3557s00.htm

Preedy, R. (2015). Conozca sus "ABC" de NIIF: Z es para Zoológicos

...no está en el alcance de la NIC 41, pero ¿qué es? , p. 5-6 PWC. Obtenido de https://www.pwc.com/ia/es/publicaciones/noticias-niif/assets/noticias-niif-mayo-2015.pdf

Portafolio. (2014). Cuarto productor de aceite en palma en el mundo obtenido de http://www.portafolio.co/economia/finanzas/colombia-cuarto-productor-aceite-palma-mundo-59140

República de Colombia. (29 de diciembre de 1993). Decreto 2649 de 1993. por el cual se reglamenta la contabilidad en general y se expiden los principios o normas de contabilidad generalmente aceptados en Colombia. Colombia. Obtenido de https://niif.com. co/decreto-2649-1993/

Rodríguez García, D. M., \& Ruiz Torres, J. C. (2016). Comparación del tratamien- to contable y financiero de la NIC 41 agricultura- NIIF para pymes, sección 34: actividades especiales y el decreto 2649 de 1993. In Vestigium Ire, 10(1), 180-207. Recuperado a partir de http://revistas.ustatunja.edu.co/index. php/ivestigium/article/view/1187

Sarmiento, D., \& CTCP. (2014). Documento de Orientación Técnica 001 - Contabilidad bajo los nuevos marcos técnicos normativos, obtenido de http://www.comunidadcontable.com/ BancoMedios/Documentos\%20PDF/ doc_ctcp_458.pdf

Superintendencia de Industria y Comercio. (2016). solicitudes de certificación proceso de palma. Obtenido de https://www.sic.gov.co/node/20661

Villacrés M.X., Aplicación de la NIC 41 (Agricultura) en la finca productora de Palmito "La Unión", ubicada en la provincia de Pichincha, Cantón Pedro Vicente Maldonado, Sector Paraíso Escondido. Quito. 2013. Pontificia Universidad Católica del Ecuador. Obtenido de http://repositorio.puce.edu.ec/ handle/22000/5693 